\title{
NoTe
}

\section{Attainment of glycaemic goals by step-up therapy with biphasic insulin aspart-70/30 in Japanese type 2 diabetic patients}

\author{
Yasuhiro Hosoi $^{1)}$, Ken-Ichi Ohtani ${ }^{1)}$, Hiroyuki Shimizu ${ }^{2)}$, Kouji Kashima ${ }^{3)}$, Noriyuki Sato ${ }^{4)}$, \\ Hitoshi Akiyama $^{5)}$ and Masatomo Mori ${ }^{6)}$ \\ 1) Department of Internal Medicine, Isesaki Municipal Hospital, Isesaki, Japan \\ ${ }^{2)}$ Faculty of Health Care, Kiryu University, Midori, Japan \\ 3) Department of Internal Medicine, Kiryu Kosei General Hospital, Kiryu, Japan \\ 4) Sato Clinic, Gunma, Japan \\ 5) Department of Internal Medicine, Toho Hospital, Midori, Japan \\ 6) Department of Medicine and Molecular Science, Gunma University Graduate School of Medicine, Maebashi, Japan
}

\begin{abstract}
We have made step-up titration protocol with biphasic insulin aspart-70/30 (BIAsp 30), and tried to achieve glycemic goals in poorly controlled Japanese type 2 diabetic patients. We summarized all results obtained to analyze the effectiveness of our protocol. The target of glycaemic control was defined as HbAlc over $7.0 \%$. In our insulin initiation protocol, all patients started a once-daily injection of BIAsp 30 before the breakfast in addition to their oral hypoglycaemic agents. The patients who could not achieve the target from 12 to 16 weeks after the start of insulin treatment proceeded to twice daily insulin injection before breakfast and dinner. Next, the patients who could not achieve the target from 12 to 16 weeks after the addition of another BIAsp injection proceeded to thrice daily insulin injection before each meal a day. The results of 39 patients were analyzed, and $10.3 \%$ of all patients achieved the target after the start of once daily injection of BIAsp 30, $41.7 \%$ achieved in twice daily injection of BIAsp, and $51.4 \%$ achieved in thrice daily injection of BIAsp. Daily insulin dose at the end of each treatment was $9.3 \pm 4.1 \mathrm{U}$ in once daily, 17.4 $\pm 6.3 \mathrm{U}$ in twice daily, and $28.4 \pm 10.4 \mathrm{U}$ in thrice daily. Total body weight increase by $2.0 \pm 2.6 \mathrm{~kg}$. The initiation and titration protocol with BIAsp 30 improved glycaemic control, and increased the number of patients with the achievement of glycaemic goals.
\end{abstract}

Key words: Biphasic insulin aspart-70/30, HbA1c, Type 2 diabetes

EVIDENCES are accumulating that rigorous glycaemic control with intensive insulin therapy delays the progression of complications in both type 1 and type 2 diabetes [1, 2]. Premix insulin analog is appropriate for the initiation and titration treatment with insulin to achieve the target of HbA1c in poorly controlled type 2 diabetic patients [3]. Using biphasic insulin aspart-70/30 (BIAsp 30), even once daily, may allow some patients to reach glycaemic targets with a degree of convenience and tolerability [4]. The 1-2-3 study showed the usefulness of step-up therapy with BIAsp

Received Oct. 15, 2010; Accepted Nov. 30, 2010 as K10E-307

Released online in J-STAGE as advance publication Dec. 21, 2010

Correspondence to: Hiroyuki Shimizu, M.D., Ph.D., Faculty of Health Care, Kiryu University, 606-7 Azami Kasagake-machi, Midori, Gunma 379-2392, Japan. E-mail: shimizu-hi@kiryu-u.ac.jp
30 in type 2 diabetic patients in the United States [5]. Based upon this clinical study, we have made clinical protocol of step-up titration treatment with BIAsp in addition to oral anti-diabetic drugs (OADs) for poorly controlled type 2 diabetic patients in multiple hospitals and clinics in Gunma, and tried to improve glycaemic control and to achieve glycaemic goals by titration of BIAsp 30 as our routine clinical practice. However, the issue whether this titration method is appropriate for the improvement of poorly controlled Japanese type 2 diabetic patients has not been confirmed yet. We summarized the results obtained, and retrospectively analyzed the effectiveness of our protocol of step-up titration therapy with BIAsp in poorly controlled Japanese type 2 diabetic patients. 


\section{Subjects and Designs}

\section{Subjects}

All subjects were poorly controlled out-patients with Japanese type 2 diabetes mellitus continuously visiting to our hospitals and clinics in Gunma Prefecture, Japan. Poorly glycaemic control was assessed by $\mathrm{HbA} 1 \mathrm{c}>8.0 \%$. We analyzed the data obtained from 39 patients who started to improve glycamic control by our step-up titration protocol with BIAsp injection from Jan 13, 2007, and finished follow-up by March 31, 2010 (20 males and 19 females; age: 62.2 \pm 8.2 yearold, BMI: $23.6 \pm 3.8 \mathrm{~kg} / \mathrm{m}^{2}$ ).

\section{Study}

This is an observational study conducted with the Declaration of Helsinki and Good Clinical Practice guidelines in 5 separate hospitals and clinics in Gunma, Japan. Insulin therapy was started based upon the informed consent of each patient. Our clinical protocol by step-up titration with BIAsp was as shown in Fig. 1. First, poorly controlled type 2 diabetic patients started a once-daily injection of BIAsp 30 before the breakfast in addition to their OADs regimen. Although initial dose of insulin was defined as $0.05 \mathrm{U} / \mathrm{kg}$, but an increase of insulin dose after initiation was basically left to the discretion of each physician, and the insulin dosage was increased by the decision of each physician to achieve the target of $\mathrm{HbA} 1 \mathrm{c}$ below $7.0 \%$. OADs continued after the start of insulin injection, but the dose of OAD may be reduced according to the decision of each physician. The patients who could not achieve the target from 12 to 16 weeks after the start of insulin treatment proceeded to twice daily insulin injection before breakfast and dinner according to the consent of each patient. Next, the patients who could not achieve the target from 12 to 16 weeks after the addition of another BIAsp injection in the breakfast proceeded to thrice daily insulin injection before each meal a day according to the consent of each patient. Data was collected in the patients who did not give their consent to add another injection of BIAsp stopped data follow-up after the end of each period. Finally, the data at 12 to 16 weeks after the start of thrice daily insulin therapy was collected to analyze the data, besides the patients who rejected step-up of insulin injection.

On the initiation of insulin therapy, patients were taught how to recognize the signs and symptoms of hypoglycaemia. When patients recognize the signs or

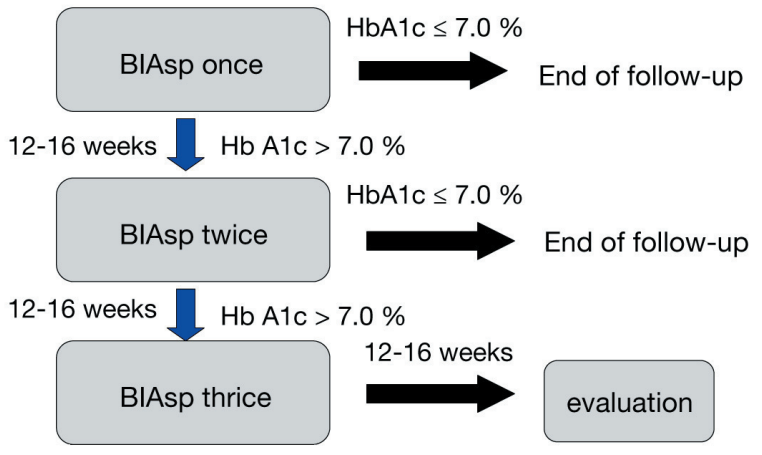

Fig. 1 Clinical protocol by step-up titration with BIAsp in the present study.

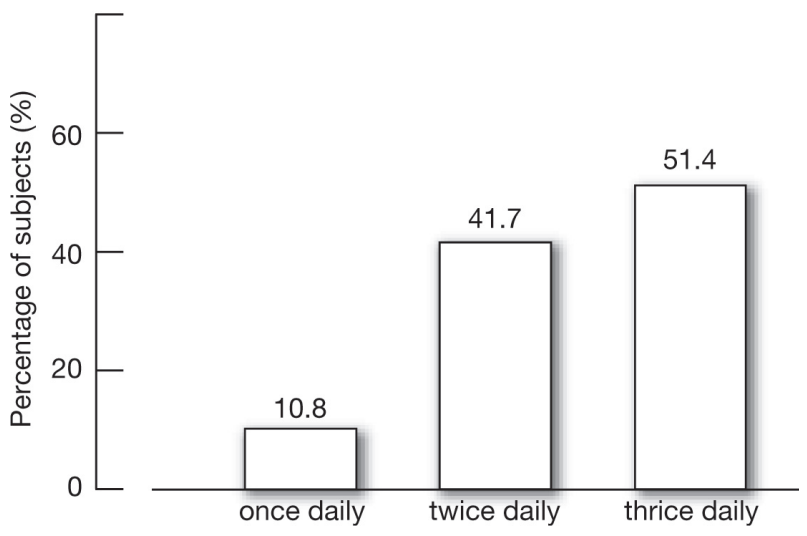

Fig. 2 Cumulative percentage of patients who achieved HbA1c $<7.0 \%$ in each treatment period with BIAsp insulin.

symptoms, patients were taught to immediately take 10-20 g sugars or glucose. Severe hypoglycaemia in the study was defined as the patients could not manage hypoglycaemia by themselves alone, and needed help of other persons.

\section{Data analysis}

Data are expressed as mean $\pm \mathrm{SD}$. All data were analyzed by analysis of variance, followed by paired $t$-test for the comparison of repeatedly measured HbA1c levels.

\section{Results}

As shown in Table 1, 2 patients interrupted insulin therapy within 12 weeks after the initiation, and 2 patients did not give informed consent to the step-up to twice daily injection, 5 patients to thrice daily injection. Fig. 2 showed cumulative percentage of patients who achieved the target of glycaemic control in each treatment period. The $10.8 \%$ of all patients achieved 


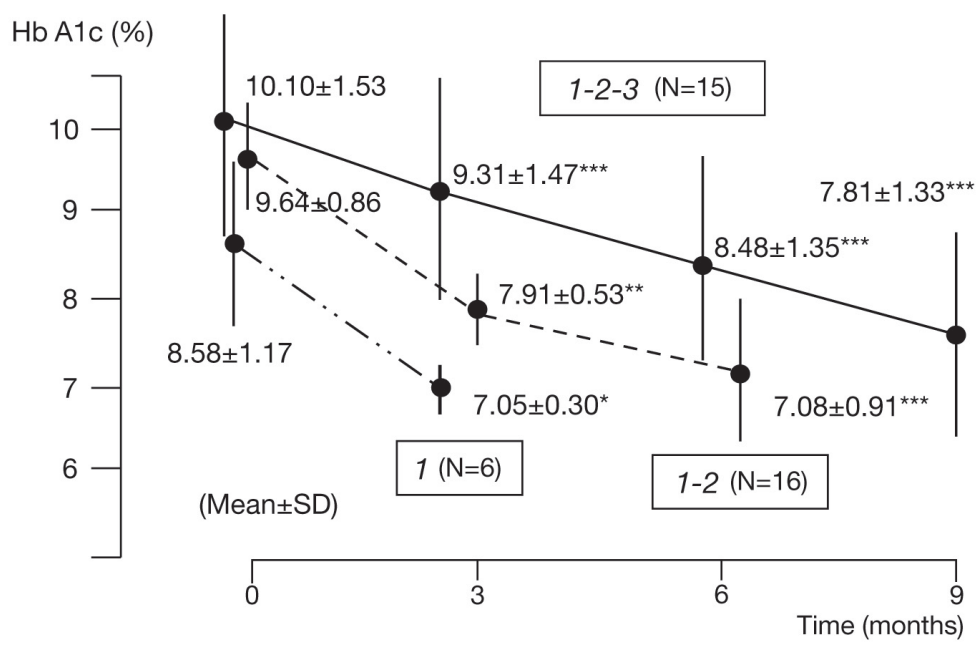

Fig. 3 Change of HbA1c levels in the patients who finished step-up titration in each treatment period of our protocol. 1: patients who achieved the target by 16 weeks or rejected to proceed twice daily BIAsp insulin injection at the step of once daily (by 12-16 weeks after the start of our protocol), 1-2: patients who achieved the target by 32 weeks or rejected to proceed thrice daily insulin injection at the step of twice daily (by 24-32 weeks after the start of our protocol), 1-2-3: patients who continued insulin injection by final step, thrice daily injection (by 36-48 weeks after the start of our protocol). Data were analyzed by paired $t$-test for the comparison of HbA1c levels in each group. *: $p<0.02, * *: P<0.005, * * *: p<0.0001$ vs. initial HbA1c in each group.

Table 1 Number of patients achieved target HbA1c and daily insulin doses in each treatment period.

\begin{tabular}{lccc}
\hline & once daily & twice daily & thrice daily \\
\hline Number of patients & 39 & 31 & 15 \\
Number of patients achieved target HbAlc $<7.0 \%$ & $4(10.80 \%)$ & $11(35.50 \%)$ & $4(26.70 \%)$ \\
Number of patients dropped & 2 & 2 & 5 \\
\hline All patients & & & \\
$\quad$ Daily insulin doses (U)** & $9.3 \pm 4.1$ & $17.4 \pm 6.3$ & $28.4 \pm 10.4$ \\
$\quad$ Daily insulin doses per body weight (U/kg body weight)** & $0.16 \pm 0.06$ & $0.28 \pm 0.10$ & $0.45 \pm 0.17$ \\
Patients achieved target HbA1c & & & \\
$\quad$ Daily insulin doses (U)* & $6.8 \pm 3.6$ & $14.0 \pm 4.3$ & $23.0 \pm 9.0$ \\
$\quad$ Daily insulin doses per body weight (U/kg body weight)* & $0.12 \pm 0.05$ & $0.24 \pm 0.12$ & $0.36 \pm 0.12$ \\
\hline
\end{tabular}

*: $p<0.005, * *: p<0.0001$ analyzed by one-way ANOVA.

the target after the start of once daily injection of BIAsp, $41.7 \%$ achieved by twice daily injection of BIAsp, and $51.4 \%$ achieved by thrice daily injection of BIAsp. There was no obvious difference in the percentage of achievement of the target among all physicians. Changes of HbA1c were shown in Fig. 3 in each treatment step, in which patients interrupted proceeding to the next step-up titration of BIAsp injection. In the patients who achieved the target by 16 weeks or rejected to proceed twice daily BIAsp insulin injection (" 1 "), HbA1c was reduced by $1.53 \%$ for 3 months. In the patients who achieved the target by 32 weeks or rejected to proceed thrice daily insulin injection (“1-2”), $\mathrm{HbA} 1 \mathrm{c}$ was reduced by $1.73 \%$ for 3 months, and 2.56
$\%$ for 6 months. Finally, in the patients who continued insulin injection by final step, thrice daily injection (" $1-2-3$ "), HbA1c was reduced by $0.79 \%$ for 3 months, $1.62 \%$ for 6 months, and $2.29 \%$ for 9 months. Initial HbA1c was lower in group " 1 ” than " $1-2-3$ ”, although there was no statistical difference.

The average of daily insulin dose increased by $9.3 \pm 4.1 \mathrm{U}(0.16 \pm 0.06 \mathrm{U} / \mathrm{kg}$ body weight $)$ at the end of once daily injection period, $17.4 \pm 6.3 \mathrm{U}(0.28 \pm 0.10$ $\mathrm{U} / \mathrm{kg}$ body weight) of twice daily, and $28.4 \pm 10.4 \mathrm{U}$ $(0.45 \pm 0.17 \mathrm{U} / \mathrm{kg}$ body weight) of thrice daily (Table 1). One-way ANOVA revealed significant differences in both daily insulin doses and daily insulin doses per body weight in all patients. In addition, daily insu- 
lin dose of patients achieved the target also increased from once daily to twice daily, thrice daily, as shown in Table 1. One-way ANOVA also revealed significant differences in both daily insulin doses and daily insulin doses per body weight in those patients. Daily insulin dose of patients achieved the target tended to be lower than all subjects included patients not achieved the target, although the differences were not statistically significant in each period. Those data indicate that the reason why the patients failed to achieve the target was not due to the short of daily insulin dose. Body weight increases by $2.0 \pm 3.1 \mathrm{~kg}$ at the end of the observation. In all patients started BIAsp titration therapy, no severe hypoglycaemia was observed in the daytime and night.

\section{Discussion}

The data obtained herein confirmed that our step-up titration protocol with BIAsp increased the number of patients achieved glycaemic control target in poorly controlled Japanese type 2 diabetic patients, and indicated that step-up titration of BIAsp is useful for the glycaemic control in Japan as well as the United States [5]. However, cumulative percentage of patients who achieved target $(\mathrm{HbA} 1 \mathrm{c}<7.0 \%)$ in the present study was obviously lower than in 1-2-3 study in the United State [5] (once daily; $11 \%$ (present study) vs. $41 \%$ (1-2-3 study), twice daily; $42 \%$ vs. $70 \%$, thrice daily; $51 \%$ vs. $77 \%$ ). Baseline HbA1c was obviously lower in the 1-2-3 study (9.6\% (present study) vs. $8.6 \%$ (1-2-3 study)). In addition, another step-up titration study of BIAsp, Sapporo 1-2-3 study, has recently reported in Japanese type 2 diabetic patients [6]. The comparison of cumulative percentage of patients who achieved target between present $(7.0 \%)$ and Sapporo $1-2-3$ (6.5 \% (JDS)) study was as follows: once daily; $11 \%$ (present study) vs. $5 \%$ (Sapporo 1-2-3 study), twice daily; $42 \%$ vs. $21 \%$, thrice daily; $51 \%$ vs. 28 $\%$ ), and baseline HbA1c of Sapporo 1-2-3 study was $8.9 \%$ (JDS). One of the reasons why cumulative percentage of patients who achieved target was obviously higher in 1-2-3 study than both present and Sapporo 1-2-3 studies may be due to the difference of baseline HbA1c on the initiation of insulin therapy. The average of baseline HbA1c on the initiation of insulin therapy has been shown to be over $9 \%$ in Japan, as shown in DAWN JAPAN study. Early initiation of insulin therapy should be necessary for achieving the target in poorly controlled type 2 diabetic patients.

Mean daily total insulin dose at the completion of each step was higher in the 1-2-3 study than present study; once daily; $0.60 \mathrm{U} / \mathrm{kg}$ body weight (1-2-3 study) vs. $0.16 \mathrm{U} / \mathrm{kg}$ body weight (present study), twice daily; $1.15 \mathrm{U} / \mathrm{kg}$ body weight vs. $0.28 \mathrm{U} / \mathrm{kg}$ body weight, thrice daily; $1.53 \mathrm{U} / \mathrm{kg}$ body weight $v s .0 .45 \mathrm{U} / \mathrm{kg}$ body weight. Mean daily total insulin dose at the completion of each step in the present study was almost same as Sapporo 1-2-3 study; once daily; 9.3 U (present study) vs. 9.8 U (Sapporo 1-2-3 study), twice daily; $17.4 \mathrm{U}$ vs. $19.3 \mathrm{U}$, thrice daily; $28.4 \mathrm{U}$ vs. $26.2 \mathrm{U}$. Patients needed higher insulin dose in the 1-2-3 study than in those two Japanese studies. This may be attributable to the difference of insulin sensitivity in type 2 diabetic patients included in each study, because the average of BMI was obviously higher in the 1-2-3 study (34 kg/ $\mathrm{m}^{2}$ ) than present study $\left(23.6 \mathrm{~kg} / \mathrm{m}^{2}\right)$ and Sapporo $1-2-3$ study $\left(25.2 \mathrm{~kg} / \mathrm{m}^{2}\right)$, indicating the existence of insulin resistance in the patients recruited in the 1-2-3 study.

As shown in 1-2-3 study and Sapporo 1-2-3 study, initiation of premix insulin injection before the dinner has a merit that it may supplement the lack of both postprandial and basal insulin secretion after the dinner by early morning, and keep adequate insulin concentrations during the night. However, it may be afraid that on starting insulin treatment, the initiation and titration of premix insulin injection before the dinner may increase the risk of nocturnal hypoglycemia by intermediate acting insulin. To avoid the risk of nocturnal hypoglycaemia from the initiation step, insulin therapy was started in the morning once a day in our treatment protocol, although clinical usefulness of the start of once daily BIAsp before the breakfast has not been well established yet. By analyzing the present data, we could demonstrate the effectiveness of the start of step-up titration of BIAsp from once daily injection before the breakfast. However, since the present results are not from randomized study, and this study did not compare the protocol with other insulin initiation and titration protocols, the results obtained herein should be limited. Further randomized study in larger population should be necessary to finally conclude the clinical usefulness of our insulin initiation and titration protocol in Japanese type 2 diabetic patients. 


\section{References}

1. Diabetes Control and Complications Trial Research Group (1993) The effect of intensive treatment of diabetes on the development and progression of long-term complications in insulin-dependent diabetes mellitus. $N$ Engl J Med 329: 977-986.

2. UK prospective Diabetes Study Group (1998) Intensive blood-glucose control with sulfonylureas or insulin compared with conventional treatment and risk of complications in patients with type 2 diabetes (UKPDS 33). Lancet 352: 837-853.

3. Fonseca V, Davidson J, Home P, Snyder J, Jellinger P, Dyhr Toft A, Barnett A (2010) Starting insulin therapy with basal insulin analog or premix insulin analog in T2DM: a pooled analysis of treat-to-target trials. Curr Med Res Opin 26: 1621-1628.
4. Halimi S, Raskin P, Liebl A, Kawamori R, Fulcher G, Yan G (2005) Efficacy of biphasic insulin aspart in patients with type 2 diabetes. Clin Ther 27 (Suppl B): S57-S74.

5. Garber AJ, Wahlen J, Wahl T, Bressler P, Braceras R, Allen E, Jain R (2006) Attainment of glycaemic goals in type 2 diabetes with once-, twice, or thrice-daily dosing with biphasic insulin aspart 70/30 (The 1-2-3 study). Diab Obes Metab 8: 58-66.

6. Yoshioka N, Kurihara Y, Manda N, Komori K, Kato M, Kijima H, Wada N, Yanagisawa K, Aoki S, Ono Y, Koike T (2009) Step-up therapy with biphasic insulin aspart-70/30-Sapporo 1-2-3 study. Diab Res Clin Pract 86: 47-52. 